\title{
Planung und Vorbereitung von Eingriffen an Ober- und Unterschenkel
}

\author{
Philipp Lichte, Hans-Christoph Pape
}

\section{Zusammenfassung}

Für den Erfolg einer Osteosynthese spielt eine gute Planung und Vorbereitung eine wichtige Rolle. Dies beginnt mit der Wahl des zu verwendenden Implantats und Zeitpunkts der Operation. Für die Entscheidungsfindung ist hierbei nicht nur die Analyse der Fraktur, sondern auch die Beachtung zahlreicher anderer Einflussfaktoren wichtig. Für das Verständnis und die Mitarbeit des Patienten ist eine sorgfältige Aufklärung über die spezifischen Risiken und Chancen des geplanten Osteosyntheseverfahrens hilfreich. Einen großen Anteil am Operationsergebnis hat die Auswahl und korrekte Durchführung der Lagerungstechnik. Insbesondere bei geschlossenen Repositionen kann sie für den Erfolg mit entscheidend sein.

\section{Einleitung}

Die Planung der Versorgung von Frakturen des Ober- und Unterschenkels hängt von verschiedenen Faktoren ab.

Hierbei spielen nicht nur frakturbedingte Einflüsse eine Rolle (Weichteilschwellung, Frakturmorphologie, offene/geschlossene Fraktur), sondern auch die aktuellen Versorgungsressourcen (Nacht, Wochenende) und gerade bei mehrfach verletzten Patienten der Allgemeinzustand und die Begleitverletzungen.

Der behandelnde Arzt sollte daher unter Berücksichtigung all dieser Faktoren eine Entscheidung bez. seines Vorgehens treffen.

OP-JOURNAL 2011; 27: 70-79

(c) Georg Thieme Verlag KG Stuttgart · New York DOI http://dx.doi.org/10.1055/s-0030-1270961

\section{Planning and Preparation for Oper- ations on the Upper and Lower Leg}

Good planning and preparation are essential prerequisites for a successful osteosynthesis. This starts with the choice of the implant to be used and the timing of the operation. Not only an analysis of the fracture but also consideration of numerous other influencing factors are important for the decision-making process. A comprehensive explanation of the specific risks and changes of the planned osteosynthetic procedure is helpful to assure the patient's understanding and cooperation. A major factor contributing to the success of the operation is the choice and correct application of the positioning technique. Especially for closed repositioning this can be decisive for success.

\section{Diagnostik}

Für die Planung der operativen Versorgung sind konventionelle Röntgenbilder des kompletten Femurs in 2 Ebenen notwendig. Um Frakturausläufer auszuschließen, sollten im Falle von proximalen oder distalen Frakturen außerdem zusätzlich Zielaufnahmen des angrenzenden Gelenks vorliegen.

Für die Planung von Revisionseingriffen bei Rotationsfehlstellungen ist die Durchführung eines präoperativen RotationsCT dringend zu empfehlen, da nur dieses eine reproduzierbare Messung ermöglicht.

\section{Verfahren}

Für die Versorgung von Frakturen des proximalen Fünftels des Schaftes wie auch der meisten subtrochantären Frakturen bieten Nagelsysteme mit Schenkelhalsklinge oder -schraube aus biomechanischen Gesichtspunkten Vorteile gegenüber extramedullären Plattensystemen.

Der folgende Artikel soll einige Entscheidungshilfen für die Auswahl der Osteosyntheseverfahren, notwendige Punkte der Patientenaufklärung und praktische Hinweise zur Lagerung bei Frakturen des Ober- und Unterschenkels bieten.

\section{Femurschaft}

Bei der Versorgung von Femurschaftfrakturen hängt die Wahl des Implantats und der Lagerung stark von der Lokalisation ab. Frakturen des proximalen Fünftels, zu denen auch die subtrochantären Frakturen gehören, werden im Regelfall wie proximale Femurfrakturen behandelt.

Die Frakturen des distalen Fünftels werden aufgrund der Versorgungsstrategie und Lagerung im Abschnitt distaler Femur beschrieben.

Dies ist insbesondere für ältere Patienten zu beachten, die deutlich von einer belastungsstabilen Versorgung und der dadurch möglichen Frühmobilisation profitieren.

Durch den Einsatz der langen Nagelvarianten für den proximalen Femur können auch sehr weit distal auslaufende subtrochantäre Frakturen oder Kombinationen aus proximalen Frakturen und Schaftfrakturen versorgt werden.

Standardtherapie zur Versorgung von klassischen Femurschaftfrakturen ist die antegrade Marknagelung [13].

Aufgrund der fehlenden Belastungsstabilität ist die Plattenosteosynthese beim Erwachsenen nur noch ausgewählten Indikationen vorbehalten. 
Die Marknagelung kann sowohl unaufgebohrt als auch aufgebohrt erfolgen. Bei jungen Patienten mit einem für die unaufgebohrte Nagelung zu schmalen Markraum und älteren Patienten mit weiten Markräumen sowie bei Revisionsoperationen bei Pseudarthrosen oder verzögerter Frakturheilung sollten aufgebohrte Marknägel eingesetzt werden.

Der Fixateur externe stellt im Bereich des Oberschenkels in aller Regel keine endgültige Lösung dar. Dennoch gibt es einige Indikationen:

- $3^{\circ}$ offene Frakturen

- temporäre Fixation bei polytraumatisierten Patienten

- Infektionen

\section{Spezielle Aufklärung}

Insbesondere bei komplexen Frakturen des Schaftes besteht ein erhöhtes Risiko für Achsfehlstellungen und Verkürzungen. Dies betrifft insbesondere Rotationsfehlstellungen, die nicht selten sekundär operativ revidiert werden müssen.

Bei der Aufklärung zur Nagelosteosynthese muss der Patient über die Möglichkeit, beim Einbringen des Nagels das Trochantermassiv zu sprengen, informiert werden.

\section{Bestimmung Nageldurchmesser und -länge}

Nagellänge und -durchmesser können im präoperativen Röntgenbild bestimmt werden. Für unaufgebohrte Nägel wird der Durchmesser im Markhöhlenisthmus in der a.-p. und seitlichen Projektion bestimmt. Die Messwerte der beiden Projektionen werden hierzu addiert und das Ergebnis durch 2 geteilt. Anschließend muss noch ein Vergrößerungsfaktor für das Röntgenbild abgezogen werden.

Für einige Nägel liegen Schablonen für die Bestimmung am präoperativen Röntgenbild vor. Auch hierbei muss jedoch ein Vergrößerungsfaktor für das Röntgenbild beachtet werden.

Alternativ kann intraoperativ eine Messlehre genutzt werden. Hierzu wird der Bildwandler in a.-p. Projektion eingestellt und die Lehre im Bereich des Isthmus auf den Oberschenkel aufgelegt. Der Übergang von Markraum zur Kortikalis muss bei Auswahl des korrekten Durchmessers auf beiden Seiten gerade noch zu sehen sein.

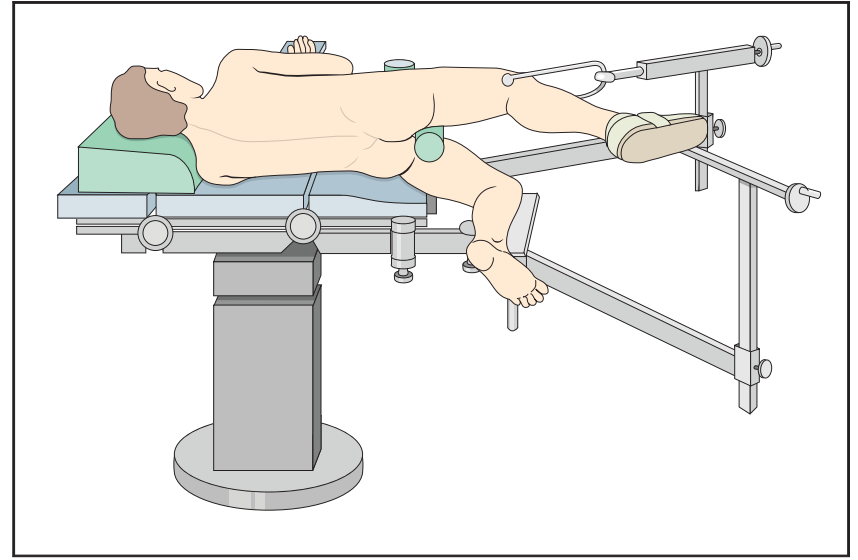

Abb. 1 Seitenlagerung des Patienten mit flektiertem Kniegelenk und zusätzlicher Nagelextension. Hierdurch wird die Reposition von Femurschaftfrakturen häufig deutlich erleichtert (modifiziert mit freundlicher Genehmigung der „AO Surgery Reference, online reference in clinical life", www.aofoundation. org).

Bei Implantation eines aufgebohrten Nagels sollte der geplante Durchmesser intraoperativ kontrolliert werden und 0,5-1,5 mm kleiner gewählt werden als der letzte Bohrkopf.

Auch für die Festlegung der Nagellänge stehen prä- und intraoperative Möglichkeiten zur Verfügung.

Da sich die Längenverhältnisse insbesondere bei komplexeren Frakturen durch Reposition jedoch deutlich ändern können, muss die Nagellänge anhand des Röntgenbilds der unverletzten Gegenseite bestimmt werden.

Soll die Längenbestimmung im OP-Saal erfolgen, kann sie mithilfe des C-Bogens und der Längenmessschablone des geplanten Nagelsystems vor dem Abwaschen und Abdecken ebenfalls an der unverletzten Gegenseite erfolgen. Hierzu ist es hilfreich, sich den gewünschten Eintrittspunkt im Trochanterbereich mithilfe des Bildwandlers zu suchen und auf der Haut zu markieren. Anschließend kann dann die Schablone lateral des Oberschenkels mit einer langen Klemme an der Markierung angelegt und nach Verschieben des Bildwandlers das distale Ende festgelegt werden. Dieses sollte etwa in Höhe der ehemaligen Epiphysenfuge liegen.

Alternativ ist mit dieser Methode natürlich auch eine Messung am verletzten Bein nach Reposition der Fraktur möglich.

Intraoperativ kann bei Nutzung standardisierter Führungsdrähte entweder durch Abmessung an einem 2. Draht oder mithilfe einer Messlehre die Nagellänge abgelesen werden.
Ist eine Kompressionsnagelung oder Dynamisierung geplant, muss der Nagel entsprechend kürzer gewählt werden, um eine Migration des Nagels zu vermeiden.

\section{Lagerung}

Die Marknagelung von Femurfrakturen kann mit oder ohne angelegte Extension durchgeführt werden. Eine sorgfältige Lagerung ist wichtig, um Rotationsfehler zu vermeiden.

Bei der Lagerung auf dem Extensionstisch ist Vorsicht geboten, um zusätzliche Dislokationen und Weichteilschäden durch Manipulationen vorzubeugen. Entscheidend ist die frühzeitige exakte Positionierung des Patienten auf dem Tisch mit direktem Kontakt zur Dammstütze sowie deren ausreichende Polsterung.

Zur Lagerung des verletzten Beines kann ein Extensionsschuh genutzt werden. Einige Autoren bevorzugen jedoch die Extension über einen suprakondylären Nagel, da hierbei der Patient mit gebeugtem Kniegelenk gelagert werden kann und durch die Entspannung der Muskulatur die Reposition erleichtert wird. Besonders in Seitenlage ist auch eine Kombination aus Extensionsschuh und suprakondylärem Nagel möglich (Abb. 1).

Für das Einbringen des Nagels in Rückenlage ist es von großem Vorteil, wenn das Becken der verletzten Seite an den Tischrand gelagert wird und der Oberkörper maximal zur gesunden Seite abgewinkelt und fixiert wird.

Ist eine Marknagelung ohne Anlage einer Extension geplant, kann diese sowohl in Rücken- als auch in Seitenlagerung erfolgen. 
Die Lagerung in Seitenlage erlaubt einen komfortableren Zugang zum Nageleintrittspunkt, jedoch ist die Kontrolle der Reposition erschwert.

In Rückenlage werden die LWS und das Sakrum mit einem Kissen unterpolstert und der Patient wiederum an den Rand des Tisches gelagert. Der Oberkörper wird zur Gegenseite abgewinkelt, um eine bestmögliche Exposition des Trochanters zu erreichen. Eine leichte Beugung in Knie und Hüftgelenk kann bei schwieriger Reposition erneut hilfreich sein.

Das unverletzte Bein wird mithilfe einer Beinschale ausgelagert, wobei auf ausreichende Polsterung des N. peroneus zu achten ist.

Vor der Auslagerung kann es zur intraoperativen Rotationskontrolle hilfreich sein, die Bewegungsausmaße für Innenund Außenrotation des Fußes am gesunden Bein zu notieren.

Ebenso kann mithilfe des Bildwandlers vor Beginn der Operation die Länge des unverletzten Oberschenkels ausgemessen werden. Als Landmarken dienen hierbei die kraniale Begrenzung des Oberschenkelkopfs sowie die laterale Gelenklinie des Knies.

Mithilfe des Bildwandlers sind weitere Methoden zur Torsionskontrolle möglich: Kortikalissprungzeichen, Trochanter-minor-Zeichen, Torsionswinkelbestimmung des Schenkelhalses mittels C-Bogen.

Für das Trochanter-minor-Zeichen wird präoperativ das gesunde Bein so gelagert, dass das Knie exakt a.-p. getroffen wird. Anschließend wird der Trochanter minor aufgesucht und dessen Stellung dokumentiert. Intraoperativ kann dann nach Rotationseinstelllung des Knies die Projektion des Trochanter minor mit der Gegenseite verglichen werden [17].

Alternativ kann vor der endgültigen Lagerung und Abdeckung eine exakt seitliche Einstellung der Kondylenebene erfolgen. Der Oberschenkel wird anschließend durch einen Assistenten in dieser Stellung fixiert. Nun wird der CBogen über dem proximalen Femur so lange durchgeschwenkt, bis Schenkelhals und Femurschaft eine Linie bilden. Der jetzt anliegende Winkel kann am C-Bogen abgelesen werden und intraoperativ das Prozedere an der verletzten Seite wiederholt werden [8].
Für die Anlage eines Fixateur externe sowie für die Plattenosteosynthese wird der Patient in Rückenlage auf einem Normaltisch gelagert, sodass das betroffene Bein frei beweglich ist. Eine Fixierung des unverletzten Beines mit einem Gurt verhindert zwar ein Verrutschen des Patienten bei Manipulation, jedoch ist dann keine Längen- und Rotationskontrolle durch das gesunde Bein möglich. Manchmal kann bei komplexen Frakturen sogar eine Freilagerung des kontralateralen Beines zur Achskontrolle sinnvoll sein.

Vor Beginn der operativen Maßnahmen sollten das Durchschwenken des C-Bogens und die überlagerungsfreie Darstellung der benötigten Regionen überprüft werden.

\section{Distale Femurfraktur}

Unter dem Sammelbegriff distale Femurfraktur werden verschiedene Frakturen subsumiert:

- suprakondyläre Frakturen (A1-A3)

- monokondyläre Frakturen (B1-B3)

- supradiakondyläre Frakturen (C1-C3)

und als Sonderform periprothetische Femurfrakturen bei einliegender Knietotalendoprothese.

\section{Diagnostik}

Standarddiagnostik ist ein konventionelles Röntgen des Kniegelenks in 2 Ebenen inkl. Patella.

Bei komplexeren intraartikulären Frakturen kann ein CT des Kniegelenks wichtige Hilfestellung bei der Planung der operativen Versorgung liefern.

\section{Verfahren}

Zur operativen Versorgung distaler Femurfrakturen stehen für die unterschiedlichen Frakturen verschiedenste Verfahren zur Verfügung:

- Zugschraubenosteosynthese

- retrograde Marknagelung

- konventionelle Plattenosteosynthese

- überbrückende Plattenosteosynthese (winkelstabile Systeme)

Suprakondyläre Frakturen können sowohl mit einem retrograden Marknagel als auch mit einer Plattenosteosynthese versorgt werden. Aus unserer Sicht ist der retrograde Femurnagel insbesondere bei einfachen Frakturen ohne Gelenkbeteiligung bei jüngeren Patienten indiziert.
Im Falle einer deutlichen Osteoporose kann ein winkelstabiles Plattensystem durch die Möglichkeit des Einbringens mehrerer Schrauben in das metaphysäre Fragment Vorteile im Sinne einer Verteilung der einwirkenden Kräfte auf eine größere Fläche bieten (Abb. 2).

Zudem können durch die Möglichkeit der minimalinvasiven Implantation zugangsassoziierte Komplikationen vermieden werden [5].

Für die Versorgung von monokondylären Frakturen und Abbrüchen der dorsalen Kondylen (Hoffa-Frakturen) bietet sich eine Zugschraubenosteosynthese an. Bei eingeschränkter Knochenqualität sollte jedoch auch der zusätzliche Einsatz einer Abstützplatte erwogen werden. Ein präoperatives CT ist hilfreich bei der Auswahl des Zugangs und der Entscheidung zwischen direkter und indirekter Verschraubung, wobei die direkte Verschraubung von dorsal nur selten durchgeführt wird. Die meisten Hoffa-Frakturen können mit 2 in a.-p. Richtung als Zugschrauben eingebrachten $6,5 \mathrm{~mm}$ Spongiosaschrauben ausreichend stabilisiert werden. Diese sind nach Möglichkeit außerhalb der femoropatellaren bzw. femorotibialen Gelenkfläche zu platzieren und die Köpfe unter Knorpelniveau zu versenken.

Supradiakondyläre Frakturen werden wie die restlichen distalen Femurfrakturen mit Gelenkbeteiligung in der Mehrheit durch winkelstabile Plattenosteosynthesen versorgt. Jedoch kann nach Rekonstruktion des Kondylenmassivs, z.B. durch Zugschrauben, auch eine retrograde Marknagelung erfolgen. Hierbei muss jedoch bedacht werden, dass ein nicht unerhebliches Risiko besteht, den Gelenkblock erneut zu sprengen. Zudem bieten die Plattensysteme den Vorteil einer größeren Variationsbreite der Schraubenlage, während die Lage der Verriegelungsbolzen beim Nagel vorgegeben ist.

Standardzugangsweg für die Plattenosteosynthese ist von lateral ventral das Septum intermusculare, der bei intraartikulärer Beteiligung bis zur Tuberositas tibia bogenförmig erweitert werden kann. Komplexere intraartikuläre Frakturen sind jedoch über den rein lateralen Zugang häufig nur schwer zu rekonstruieren. Hier kann ein relativ kleiner parapatellarer oder medial streckseitig gelegener Zugang hilfreich sein, um den Gelenkblock schrittweise zu rekonstru- 

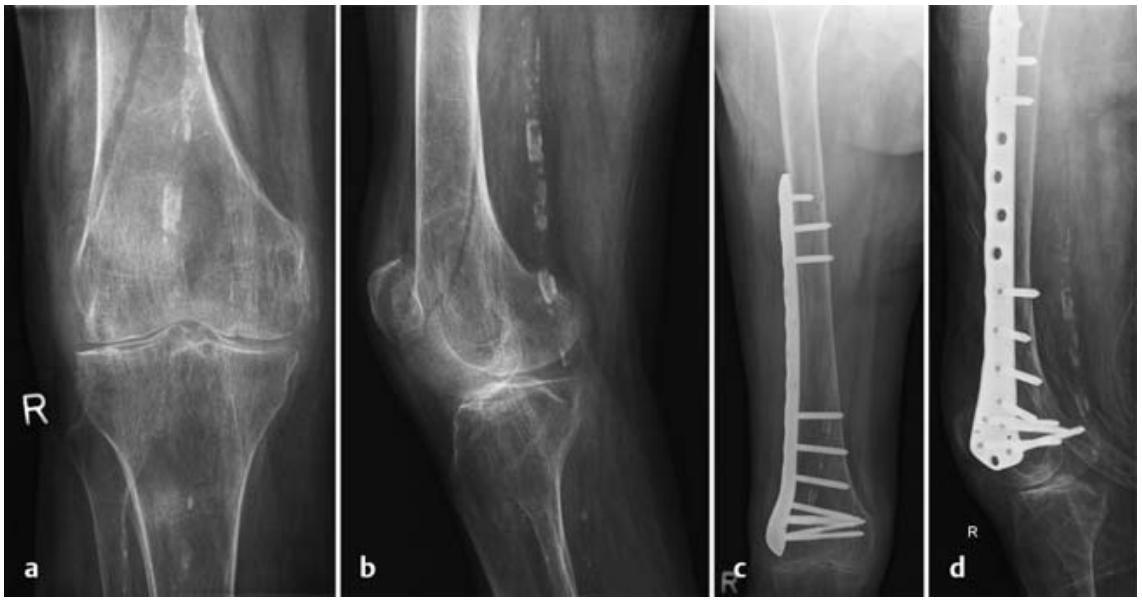

Abb. 2 a bis $\mathbf{d}$ Bei distalen Femurfrakturen mit vorbestehender Osteoporose bietet die minimalinvasive winkelstabile Plattenosteosynthese Vorteile gegenüber der Marknagelung.

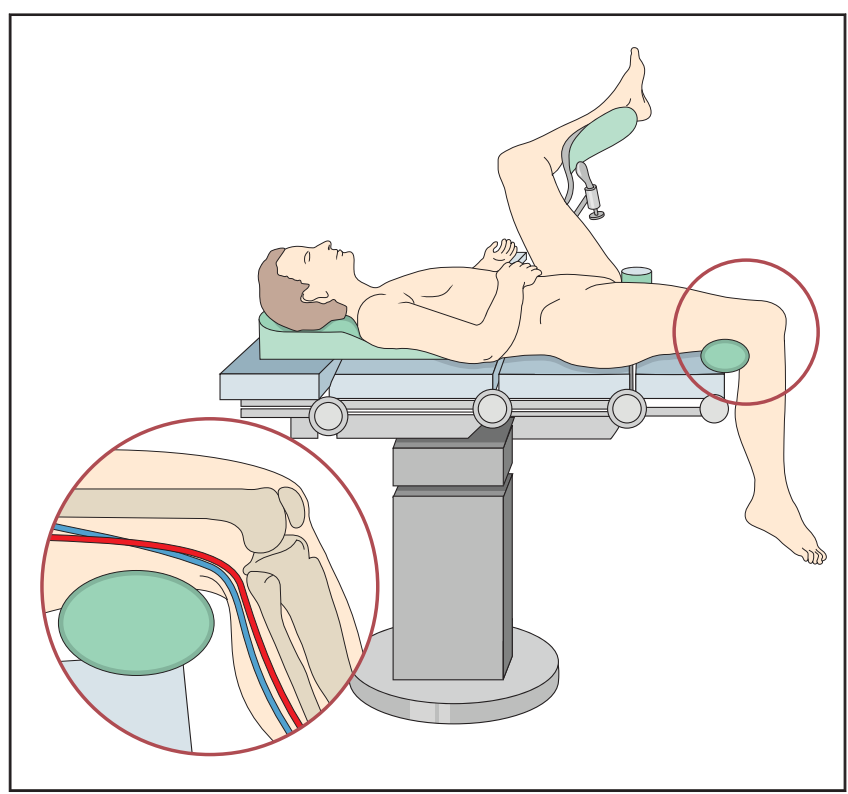

Abb. 3 Für die retrograde Marknagelung kann das verletzte Bein über das abgeklappte oder entfernte Fußteil gelagert werden, jedoch muss auf eine kompressionsfreie Lagerung der Kniekehle geachtet werden (modifiziert mit freundlicher Genehmigung der „AO Surgery Reference, online reference in clinical life“, www. aofoundation.org).

ieren. Anschließend kann über einen gesonderten Zugang eine durchgeschobene Platte zur Stabilisierung eingebracht werden.

Insbesondere für die Versorgung von supradiakondylären Frakturen ist eine präzise präoperative Planung von großer Bedeutung und kann den OP-Ablauf deutlich beschleunigen.

Für die gängigen Implantate liegen Schablonen vor, mit deren Hilfe anhand der gesunden Gegenseite Implantatund Schraubenlage geplant werden können. Insbesondere sollte frühzeitig die Notwendigkeit der Spongiosaplastik überdacht werden, da der Patient hierüber aufgeklärt werden und die Lagerung entsprechend angepasst werden muss.

\section{Nagellänge retrograder Femurnagel}

Bei der Längenplanung für einen retrograden Femurnagel sollte die Position der proximalen Verriegelungsschrauben beachtet werden: 4-6 $\mathrm{cm}$ unterhalb des Trochanter minor befindet sich eine Region, in der die A. femoralis und Äste des N. femoralis stark gefährdet sind.

Daher sollten Verrieglungsschrauben in dieser Region vermieden werden und der Nagel lieber so lang gewählt werden, dass die Verriegelungen unterhalb der kritischen Region oder oberhalb des Trochanter minor zu liegen kommen.

\section{Lagerung}

Der Patient wird in Rückenlage mit vorbereiteter Oberschenkelblutsperre auf einem röntgendurchlässigen Tisch gelagert. Um den Einsatz des C-Bogens in beiden Ebenen zu ermöglichen, wird das unverletzte Bein in $90^{\circ}$-Beugung in Hüfte und Kniegelenk ausgelagert. Hierdurch kommt es allerdings zu einer Verkippung des Beckens. Daher muss in der Folge beachtet werden, dass das proximale Fragment der frakturierten Seite um ca. $20^{\circ}$ nach außen rotiert zu liegen kommt.

Zur Erleichterung der Reposition durch Neutralisation des Gastroknemiuszugs kann das frakturierte Bein im Kniegelenk in $90^{\circ}$-Beugung gelagert werden.

Hierdurch wird eine Fehlstellung in der sagittalen Ebene häufig schon adäquat reponiert. Auch das Auffinden des korrekten Eintrittspunkts wird so leichter. Technisch gibt es verschiedene Möglichkeiten: Der Oberschenkel kann bei abgeklapptem Beinteil über eine Rolle gelagert und der Unterschenkel frei hängen gelassen werden (Abb.3). Eine andere Möglichkeit bietet die Nutzung des Arthroskopiebeinhalters. Andere Autoren bevorzugen eine Lagerung mit lediglich 40-60-Beugung im Kniegelenk [9].

Auch für die Plattenosteosynthese wird eine leichte Beugung im Kniegelenk zur Neutralisation des Muskelzugs empfohlen. Hierzu wird der Patient in Rückenlage mit dem Kniegelenk exakt über dem Gelenk der manuellen Beinplattenverstellung gelagert. Das distale Beinteil wird dann manuell um einige Grad nach unten abgeknickt. In dieser Stellung wirkt die kurze Beinplatte als Hypomochlion und die Reposition v.a. von suprakondylären Frakturen wird durch die Schwerkraft vereinfacht.

\section{Tibiakopffrakturen}

\section{Diagnostik}

Zusätzlich zur konventionellen Röntgenaufnahme des Kniegelenks in 2 Ebenen ist eine Computertomografie mit 2-Doder 3-D-Rekonstruktionen zur OP-Planung dringend zu empfehlen. Mit ihrer Hilfe wird die Zuordnung der Fragmente deutlich erleichtert und Defektzonen mit der Notwendigkeit des Auffüllens mit Knochen- oder Knochenersatzmaterial aufgedeckt. In komplexen Fällen können Röntgenaufnahmen der unverletzten Gegenseite sinnvoll sein, um die physiologische Gelenkstellung zu analysieren und die spätere Implantatlage mittels Schablonen zu planen. 
Bei Luxationsfrakturen ist neben der klinischen Kontrolle der Fußpulse eine Gefäßdarstellung indiziert, da es nicht selten zu Verletzungen der A. poplitea kommt.

Hier bietet sich das Kontrastmittel-CT an. Auch ist bei klinischen Zweifeln eine Kompartmentdruckmessung zu empfehlen, da das Risiko für ein Kompartmentsyndrom insbesondere bei Luxationsfrakturen deutlich erhöht ist.

Ein MRT kann Hinweise auf Kniebinnenschäden wie Band- oder Kapselverletzungen liefern.

\section{Verfahren}

Ziel der operativen Therapie ist eine Wiederherstellung der Kongruenz der Gelenkflächen im Bereich der Belastungszonen des Tibiaplateaus sowie eine achsgerechte Stellung des Kniegelenks. Hierzu stehen primär

- Zugschraubenosteosynthesen

- Plattenosteosynthesen

- Fixateur externe inklusive Hybridfixateur

zur Verfügung.

Extraartikuläre Frakturen (A2 und A3) können mit Zugschrauben und Abstützplatten oder in zunehmendem Ausmaß mit winkelstabilen Implantaten von lateral versogt werden.

Reine Spaltfrakturen (B1) können mit 2 Zugschrauben mit Unterlegscheiben gering invasiv versorgt werden. Hierbei ist jedoch die Knochenqualität zu beachten, da bei ausgeprägter Osteoporose eine zusätzliche Abstützplatte sinnvoll sein kann. Auch für B3-Frakturen kann ein ähnliches Vorgehen angewendet werden, jedoch ist hierbei die imprimierte Gelenkfläche anzuheben und ggf. mit Knochenmaterial zu unterfüttern. Die Abstützung erfolgt, wie auch nach der Anhebung der Gelenkfläche bei reinen Impressionsfrakturen (B2), durch subchondrale Spongiosaschrauben.

Zur Beurteilung der Gelenkfläche kann eine arthroskopische Unterstützung der Osteosynthese hilfreich sein.

Bei höhergradigen Frakturen wird der Stellenwert der Arthroskopie kontrovers diskutiert $[3,19]$. Bei Vorliegen von Verletzungen der Kapsel besteht jedoch ein hohes Risiko für ein sekundäres Kompartmentsyndrom durch Austritt der Spülflüssigkeit mit hohem Druck in das umliegende Gewebe.
Der optimale Zeitpunkt der operativen Versorgung wird kontrovers diskutiert. Während einige Arbeiten eine sofortige Versorgung innerhalb der ersten 6 Stunden empfehlen, bevorzugen andere Autoren eine verzögerte Versorgung innerhalb der 1 . Woche, um postoperative Wundheilungsstörungen zu vermeiden.

Gradmesser für die Zeitplanung sollte immer die Weichteilsituation sein. Für komplette Gelenkfrakturen (C1-C3) sind aufgrund der massiven Weichteilschädigung und des komplexen Eingriffs eher eine primäre Ruhigstellung mit gelenküberbrückendem Fixateur externe und eine sekundäre definitive Versorgung zu empfehlen.

Implantate der Wahl für diese Frakturen sind winkelstabile Plattensysteme, die von lateral eingebracht werden [12]. Diese schonen durch die Möglichkeit der teilweise perkutanen Schraubenbesetzung die Weichteile. Ist präoperativ abzusehen, dass eine allein laterale Versorgung nicht ausreicht und eine additive mediale Versorgung mit Schrauben oder Abstützplatte geplant ist, so müssen die Zugänge aufeinander abgestimmt werden. Der Abstand sollte, um eine ausreichende Durchblutung zu gewährleisten, mindestens $6 \mathrm{~cm}$ betragen.

Eine Alternative bietet bei komplexen Situationen und schwerem Weichteilschaden die Anlage eines Hybridfixateurs, ggf. in Kombination mit einzelnen Spongiosaschrauben zu Unterstützung der Gelenkfläche.

\section{Spezielle Aufklärung}

Wie bei allen intraartikulären Frakturen muss der Patient über das erhöhte Risiko einer posttraumatischen Arthrose informiert werden. Je nach operativem Verfahren ist insbesondere der N. peronaeus gefährdet. Des Weiteren können im Verlauf ergänzende Eingriffe zur Rekonstruktion des Bandapparats notwendig sein. Insbesondere bei ausgeprägtem Weichteilschaden und arthroskopischer OP-Unterstützung besteht ein hohes Risiko für ein Kompartmentsyndrom.

\section{Lagerung}

Die Lagerung erfolgt in Rückenlage mit prophylaktisch angelegter Blutsperre. Der Unterschenkel muss zur Kontrolle der Achsenverhältnisse frei gelagert werden. Wir empfehlen bei komplexeren Frakturen immer, den Beckenkamm mit vorzubereiten, um im Zweifelsfall Zugriff auf autologe Spongiosa zu haben.

Das Kniegelenk sollte wie bei der Osteosynthese des distalen Femurs im Kniegelenk leicht gebeugt gelagert werden.

Dies kann ebenfalls durch eine geringe Abknickung der Beinplatte von 3-5 Rasten erreicht werden. Auch hierbei ist entscheidend, dass das Knie direkt oberhalb des Scharniers zu liegen kommt. Im Unterschied zur Versorgung distaler Femurfrakturen sollte der Tisch jedoch anschließend so gekippt werden, dass der Unterschenkel waagerecht liegt. Die Beugung im Kniegelenk ist natürlich auch durch Lagerung auf einer Rolle oder einem Kissen im Kniebereich möglich, jedoch bietet die o.g. Variante eine höhere Stabilität und eine verbesserte Orientierung bei waagerechter Unterschenkellage durch die Tischkippung.

Bei der Lagerung des unverletzten Beines ist die überlagerungsfreie Durchleuchtung in beiden Ebenen zu beachten. Daher kann eine Auslagerung mit gebeugtem Hüftgelenk in einem Beinhalter sinnvoll sein. Alternativ kann das unverletzte Bein komplett abgesenkt werden, bis es außerhalb des seitlichen Strahlengangs liegt.

\section{Unterschenkelschaftfrakturen}

\section{Diagnostik}

Für die OP-Planung reichen meist konventionelle Röntgenaufnahmen im a.-p. und seitlichem Strahlengang. Wichtig ist jedoch, dass die angrenzenden Gelenke Knie und oberes Sprunggelenk vollständig und eindeutig dokumentiert sind. Bei der Planung nicht bedachte metaphysäre Ausläufer von Frakturen können ansonsten das geplante Verfahren unmöglich machen oder im Verlauf $\mathrm{zu}$ einem Osteosyntheseversagen führen.

\section{Verfahren}

Das Standardosteosyntheseverfahren für Frakturen des Tibiaschafts ist die Marknagelung.

Durch die Entwicklung der modernen Verriegelungsnägel mit Winkelstabilität und weit distal gelegenen Verriegelungsmöglichkeiten hat sich das Einsatzgebiet deutlich erweitert. Indikationen für die Marknagelosteosynthese sind

- kurze Schaft- und Querfrakturen im mittleren Drittel des Schaftes

- erst- bis zweitgradig offene Frakturen 
- Etagenfrakturen

- Frakturen mit höhergradigem Weichteilschaden (ggf. nach Primärversorgung im Fixateur externe)

- instabile Hochenergieverletzung, insbesondere bei polytraumatisierten $\mathrm{Pa}$ tienten mit beidseitigen Verletzungen

Tibianägel können sowohl in aufgebohrter als auch in unaufgebohrter Technik eingebracht werden. Ob eine Methode der anderen überlegen ist, wird derzeit kontrovers diskutiert. Die aufgebohrte Technik erlaubt es jedoch, auch bei enger Markhöhle Nägel mit größerem Durchmesser und damit einer höheren Primärstabilität einzusetzen.

Bei Unterschenkelfrakturen der distalen Hälfte kann eine zusätzliche Stabilisierung der Fibula die Gesamtstabilität verbessern und v. a. die Reposition deutlich erleichtern. Liegt die Fibulafraktur im Bereich des distalen Fünftels, ist eine operative Versorgung obligat, da ansonsten eine korrekte Einstellung der Malleolengabel nicht gewährleistet werden kann.

Wird ein operatives Angehen der Fibulafraktur geplant, so sollte mit dieser begonnen werden, um eine korrekte Wiederherstellung der Länge und Einstellung des OSG zu erreichen.

Während durch die Marknagelosteosynthese zumindest bei einfacheren Frakturen eine belastungsstabile Versorgung möglich ist, ist die Plattenosteosynthese lediglich übungsstabil.

Indikationen für die Plattenosteosynthese sind:

- Frakturen in unmittelbarer Nähe zur Metaphyse

- kombinierte metaphysäre und diaphysäre Frakturen

- komplexe Frakturen im Bereich des proximalen Unterschenkeldrittels

Plattenosteosynthesen sollten an der Tibia nur bei guten Weichteilverhältnissen durchgeführt werden, da aufgrund der geringen Weichteildeckung der Tibia eine deutlich erhöhte Gefahr für Wundheilungsstörungen und Infektionen besteht.

Zur Auswahl stehen verschiedene Implantate, deren Einsatz v.a. von der Frakturmorphologie und der Lokalisation abhängig ist. Schräg- und Spiralfrakturen können häufig mit einer Kombination aus Zugschraube und Low Contact-Dynamic Compression Plate (LCDCP) versorgt werden. Für Trümmerfrakturen und insbesondere bei osteopenen Knochenverhältnissen bieten winkelstabile Implantate große Vorteile [1]. Diese sollten, wenn möglich, minimalinvasiv als durchgeschobene Platten eingebracht werden, um die Weichteile und die Durchblutung der Frakturregion bestmöglich zu schonen.

Der Fixateur externe stellt im Bereich der Tibia in den allermeisten Fällen nur eine vorübergehende Lösung zur Stabilisation dar. Indikationen sind:

- hochgradiger Weichteilschaden und hochgradig offene Frakturen

- primäre Inoperabilität bei polytraumatisierten Patienten

- Infektionen

In Einzelfällen kann bei ausgeprägter Trümmersituation mit großen Defektzonen die Anlage eines Ilizarov-Fixateurs zur definitiven Versorgung erwogen werden.

Aufgrund der mangelnden Stabilität, der mit fortschreitender Dauer steigenden Gefahr von Pininfekten und der häufig beobachteten Pinlockerung sollte eine definitive Versorgung mit Nagel- oder Plattenosteosynthese im Verlauf geplant und durchgeführt werden.

Schon bei Anlage des Fixateurs sollte die Lage des Zugangs sowie des Implantats zur endgültigen Osteosynthese bedacht werden, um eine Kontamination des entsprechenden Gebiets zu vermeiden.

\section{Spezielle Aufklärung}

Der Patient muss bei allen Frakturen des Unterschenkels explizit über das Risiko der Entstehung, die Symptome sowie die dann notwendigen operativen Maßnahmen bei einem Kompartmentsyndrom aufgeklärt werden. Der Patient sollte zudem darauf hingewiesen werden, dass je nach intraoperativem Befund eine primäre Faszienspaltung notwendig werden kann.

Des Weiteren sollte die Aufklärung Dreh-, Achs- und Längenfehler mit einschließen. Insbesondere bei Trümmerzonen oder größeren Defektzonen kann eine sekundäre Knochentransplantation notwendig werden.

\section{Lagerung}

Die Umlagerung und Vorbereitung des Patienten sollte unter ständiger ärztlicher Aufsicht durchgeführt werden, wobei der ständigen Extension des verletzten Beines eine wichtige Bedeutung zukommt, um Schmerzen zu lindern, weitere Weichteilschäden zu vermeiden und das Risiko für die Ausbildung eines Kompartmentsyndroms zu vermindern.

Eine Manschette für die Anlage einer Blutsperre am Oberschenkel sollte vor Abschluss der Lagerungsmaßnahmen angelegt werden.

Die klassische Lagerung für die Marknagelung der Tibia ist die Rückenlagerung ohne Extension.

Intraoperativ muss zur Eröffnung des Markraums und zum Einbringen des Nagels das Knie auf mindestens $70^{\circ}$ gebeugt werden können.

Jedoch ist die maximal erreichbare Beugung operationstechnisch günstig [9]. Eine Beugung durch Aufstellen der Ferse auf dem OP-Tisch ist jedoch nur bei stabilen Frakturen möglich.

Daher stehen verschiedene Hilfsmittel zur Verfügung. Häufig genutzt wird z.B. ein röntgendurchlässiges Karbondreieck, über welches das Kniegelenk gelagert werden kann. Hierdurch wird die Beugung im Kniegelenk gewährleistet. Zudem bewirkt das Eigengewicht des freihängenden Unterschenkels in vielen Fällen einen Repositionseffekt. Eine einfache Alternative ist ein steriles doppelseitiges Dreieck aus Fixateurkarbonstangen, dessen proximale Querstange mit mehreren Tüchern gepolstert wird.

Anstatt des Dreiecks kann auch ein am Tisch montierbarer Beinhalter verwendet werden. Ein ähnlicher Effekt kann durch Abknicken des Fußteils erreicht werden, jedoch wird bei diesem Aufbau die Gewährleitung der Sterilität und das Durchleuchten in 2 Ebenen deutlich erschwert.

Das unverletzte Bein sollte so gelagert werden, dass ein Durchschwenken des C-Bogens in beiden Ebenen möglich ist. Hierzu kann es entweder in einer Gynschale in Flexion und Abduktion ausgelagert werden oder das entsprechende Beinteil soweit abgesenkt werden, dass eine seitliche Aufnahme möglich ist. 
Auch die Marknagelung der Tibia ist in Extension möglich. Hierbei wird der Fuß entweder über eine kalkaneare Extension oder mit Klebebändern und Binden fixiert. Eine Schuhextension ist aufgrund der Lage der distalen Verriegelungsbolzen des Nagels in den seltensten Fällen möglich. Hüfte und Knie werden jeweils auf $90^{\circ}$ flektiert, die Zugrichtung des Unterschenkels zeigt leicht nach unten, wobei eine Stütze am distalen Oberschenkel als Hypomochlion dient. Eine Kompression der Kniekehle ist unbedingt zu vermeiden [9].

Plattenosteosynthese und Fixateuranlage erfolgen in Rückenlage.

\section{Distaler US}

In diesem Abschnitt sollen Frakturen des oberen Sprunggelenks und des Pilon tibiale dargestellt werden. Aufgrund der Unterschiede im Entstehungsmechanismus und der Therapie werden diese getrennt voneinander behandelt.

\section{Frakturen des OSG}

\section{Diagnostik}

Zur OP-Planung im Bereich des OSG reichen im Regelfall konventionelle Röntgenbilder des OSG in 2 Ebenen aus.

Hierbei ist darauf zu achten, dass eine Aufnahme in korrekter Einstellung des Fußes in $20^{\circ}$-Innenrotation („Mortise View“) vorliegt [2].

Je nach Klinik sind Aufnahmen des Unterschenkels (Maisonneuve-Fraktur) zu ergänzen.

\section{Wahl des Verfahrens}

Ziel der operativen Versorgung ist die exakte Rekonstruktion der Sprunggelenksgabel. Dies erfolgt klassischerweise im Bereich des Außenknöchels mittels einer Zugschraubenosteoynthese mit Drittelrohrneutralisationsplatte. Bei Trümmerfrakturen oder Situation mit Knochenverlust sollte der Einsatz von 3,5 mm LCDC-Platten erwogen werden, da diese im Vergleich zur Drittelrohrplatte zwar etwas mehr auftragen, jedoch auch deutlich mehr Stabilität bieten.

Die Versorgung sollte in Abhängigkeit von der Weichteilschwellung innerhalb der ersten 6-8 Stunden oder 4-6 Tage nach Abschwellung in Gipsretention durchgeführt werden.
Bei massivem Weichteilschaden oder nicht im Gips retinierbaren Luxationsfrakturen kann bis zur Weichteilkonsolidierung ein Fixateur externe angelegt werden.

Im Bereich des Innenknöchels stehen zur Osteosynthese Zugschrauben und Zuggurtungen zur Verfügung. Kleinere Fragmente oder Trümmerzonen sind häufig besser mit einer Zuggurtung zu adressieren. Die Zugschrauben oder eine Kombination aus einer Schraube und einem K-Draht zur Rotationssicherung bieten dagegen den Vorteil, nach geschlossener Reposition minimalinvasiv über Stichinzisionen vorgehen zu können.

Zeigt sich in der radiologischen Diagnostik ein hinteres Volkmann-Dreieck, so sollte dieses ab einer Größe von > ein Viertel der Gelenkfläche mit Zugschrauben fixiert werden. Die Einstellung des Fragments erfolgt meist spontan nach Reposition der Malleolen in Dorsalflexion. Andernfalls ist eine offene Reposition angezeigt. Die Verschraubung erfolgt in den meisten Fällen mittels kanülierten Spongiosaschrauben über Stichinzisionen von ventral.

\section{Lagerung}

Die Lagerung des Patienten erfolgt in Rückenlage mit angelegter Oberschenkelmanschette zur Blutsperre. Bei Frakturen des Außenknöchels empfiehlt sich das Unterlegen eines flachen Keiles unter die Beckenseite des verletzten Beines. Hierdurch kippt das gesamte Bein ein wenig nach innen und der Zugang zum Außenknöchel wird erleichtert. Bei komplexen Frakturen kann es zur Rotationskontrolle hilfreich sein, bis oberhalb des Kniegelenks abzudecken, um die Patella als Landmarke nutzen zu können.

Ist eine offene Reposition des hinteren Volkmann-Dreiecks notwendig, so muss der Patient häufig in Bauch- oder Seitenlage umgelagert werden.

\section{Pilon tibiale}

\section{Diagnostik}

Pilon-tibiale-Frakturen sind Hochenergieverletzungen und daher oftmals mit einem hochgradigen Weichteilschaden assoziiert.
Zur OP-Planung ist es bei komplexeren Frakturen empfehlenswert, neben den konventionellen Aufnahmen des OSG auch ein CT mit 2-D- oder 3-D-Rekonstruktionen durchzuführen.

Dies erleichtert die Zuordnung von Fragmenten und die richtige Einschätzung des Grades der Impaktierung und des Knochenverlusts. Diese Informationen sind wichtig zur Planung, ob ggf. eine Spongiosaplastik oder Knochenersatzstoffe zur Rekonstruktion der Gelenkfläche notwendig sein werden.

\section{Verfahren}

Auf Basis einer gründlichen Untersuchung der Weichteilverhältnisse muss entschieden werden, ob eine 1-zeitige Versorgung mit primärer definitiver Plattenosteosynthese möglich ist oder ein 2-zeitiges Vorgehen mit Anlage eines Fixateur externe, ggf. Fibulaosteosynthese und der Osteosynthese der Tibia im Intervall sinnvoller erscheint.

Ein 1-zeitiges Vorgehen ist zu empfehlen bei

- einfachen Frakturen mit sicher zu klassifizierendem leichtem Weichteilschaden

- verzögerter Operation bei primär nicht operativer Ruhigstellung

Ein 2-zeitiges Vorgehen empfiehlt sich hingegen bei

- offenen Frakturen

- Frakturen mit schwerem oder nicht sicher einschätzbarem Weichteilschaden

Bei Entscheidung für ein 2-zeitiges Vorgehen sollte, wenn die lateralen Weichteile es zulassen, zuerst eine Reposition und Osteosynthese der Fibula erfolgen. Anschließend wird ein sprunggelenksübergreifender Fixteur externe mit Extension des OSG über einen kalkanearen Steinmann-Nagel angelegt.

Auch beim 1-zeitigen Vorgehen empfiehlt sich, zuerst die Fibula zu versorgen. Für die Osteosynthese der Tibia stehen verschiedene vorgeformte winkelstabile Plattensysteme zur Verfügung, alternativ können aber auch L-, T- oder DC-Platten genutzt werden. Bei der Rekonstruktion des Gelenkblockers können manchmal kanülierte Spongiosazugschrauben dazu dienen, größere Fragmente gegeneinander zu fixieren. In verbleibende Defektzonen, z.B. nach Aufstößeln der Gelenkfläche, kann Beckenkammspongiosa gestößelt werden. 
Als Alternative, insbesondere bei fortbestehend schwierigen Weichteilverhältnissen, kann eine indirekte oder minimal offene Rekonstruktion des Gelenks mit Drähten und Schrauben in Verbindung mit einem extraartikulären Ring- oder Hybridfixateur darstellen.

Zwar ist hierbei die Wiederherstellung der Gelenkfläche limitiert, jedoch sind die Infektraten niedriger als bei offenen Rekonstruktionen $[6,16]$.

\section{Spezielle Aufklärung}

Der Patient muss über das erhöhte Risiko von Wundheilungsstörungen und Infekten aufgeklärt werden. Zudem sollte er auf die lange Entlastungsphase und das hohe Risiko für die Entwicklung einer posttraumatischen Arthrose hingewiesen werden. Es empfiehlt sich außerdem, auch über die Möglichkeit der Spongiosatransplantation aufzuklären.

\section{Lagerung}

Die operative Versorgung erfolgt in Rückenlage mit angelegter Oberschenkelblutsperre. Im Zweifel sollte der Beckenkamm immer mit abgewaschen und abgedeckt werden, sodass eine Entnahme von Spongiosa möglich ist.

\section{Besonderheiten bei kindlichen Frak- turen der unteren Extremität}

Bei der Auswahl des geeigneten Verfahrens spielt neben der Frakturmorphologie und den Begleitverletzungen bei Kindern das Alter eine entscheidende Rolle [20].

Bei Heranwachsenden mit geschlossenen Wachstumsfugen kann die Versorgung analog zum Erwachsenen erfolgen.

Als Verfahren stehen bei kindlichen Frakturen zur Verfügung:

- intramedulläre Schienung mit elastischen Titannägeln (ESIN)

- Fixateur externe

- Plattenosteosynthese

Im Alter bis zu 3 Jahren können nahezu alle Frakturen des Schaftbereichs konservativ in Gipsruhigstellung behandelt werden. Für Schaftfrakturen des Oberschenkels sowie instabile Unterschenkelfrakturen bei Kindern zwischen dem 3. und ungefähr 14. Lebensjahr stellt mittlerweile die Versorgung mittels ESIN die Methode der Wahl dar [14, 15].
Ein Beispiel ist in Abb. 4 dargestellt. Im Bereich des Unterschenkels ist allerdings häufig schon bei 12-Jährigen die benötigte Stabilität nicht mehr mit ESIN allein zu erreichen.

Für Frakturen mit Trümmerzone oder langstreckige Schrägbrüche, die sich mit dem ESIN nicht ausreichend stabilisieren lassen, bietet im Unterschied zum Erwachsenen die Ausbehandlung im Fixateur externe eine Option, wobei zirkuläre Systeme eine geringere Rate an sekundären Dislokationen im Vergleich zu monolateralen Systemen zeigen $[4,7]$.

Plattenosteosynthesen werden aufgrund der Invasivität im mittleren Oberschenkelschaftbereich nur noch in Ausnahmefällen durchgeführt. Im Bereich des Unterschenkels können sie bei Frakturen, die mit ESIN nicht sinnvoll zu versorgen sind, eine gute Alternative bieten.

Subtrochantäre und metaphysäre Frakturen der unteren Extremität lassen sich in den meisten Fällen mit Plattenosteosynthesen versorgen. Hierbei muss bei der Bestimmung der Schraubenlänge auf eine konsequente Schonung der Wachstumsfuge geachtet werden.

Alternativ kann bei Frakturen im diametaphysären Übergangsbereich auch hier als minimalinvasives Verfahren häufig die ESIN-Methode eingesetzt werden, wobei für distale Frakturen eine deszendierende Nagelung zu bevorzugen ist.

Verletzungen der Epiphysenfuge werden bei ausreichend großem metaphysären Keil mit Zuschrauben versorgt. Für reine Epiphyseolysen ist die Fixation mit gekreuzten K-Drähten zu empfehlen. Allerdings muss hier supportiv auch eine Gipsruhigstellung erfolgen.

\section{Bestimmung Nageldicke ESIN}

Die Nageldicke des ESIN kann im präoperativen Röntgenbild bestimmt werden und sollte ein Drittel des Markraumisthmus betragen.

\section{Lagerung}

Zur ESIN-Versorgung des Oberschenkels hat sich die Lagerung in Rückenlage ohne Extension bewährt. Eine Versorgung in Extension ist grundsätzlich ebenfalls möglich, allerdings muss die Fraktur hierfür nach Anlage der Extension unter radiologischer Kontrolle exakt reponiert sein. Eine Blutsperre ist nicht

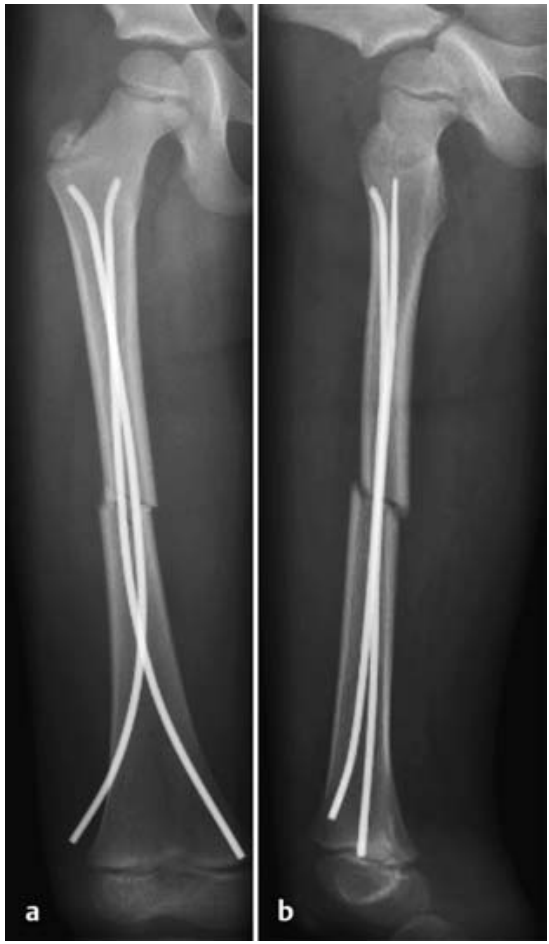

Abb. 4a und b Frakturversorgung Femurschaft mit ESIN bei 6-jährigem Jungen.

notwendig. Für die ESIN-Versorgung des Unterschenkels wird das Knie leicht gebeugt über eine Rolle gelagert. Knie und Sprunggelenk müssen intraoperativ frei zugänglich sein.

\section{Besonderheiten der OP-Planung bei mehrfachverletzten Patienten}

Bei der Versorgung von polytraumatisierten Patienten besteht ein erhöhtes Risiko einer sekundären Verschlechterung des Allgemeinzustands durch ausgedehnte operative Versorgungen. Einerseits ist die Frakturversorgung wichtig zur Schmerzreduktion, Vermeidung von Fettembolien und eine frühzeitige Mobilisation des Patienten. Hierzu eignet sich die definitve Versorgung am besten und eine Versorgung mit externen Fixateuren, Gipsverbänden oder Schienen sollte vermieden werden. Andererseits bevorzugen mittlerweile bei instabilen oder sog. „Borderline-Patienten“ viele Autoren ein 2-zeitiges Vorgehen mit primärer Stabilisierung und sekundärer endgültiger Osteosynthese $[11,18]$. Hierdurch soll der inflammatorische Stimulus durch ausgedehnte Operationen in der Primärphase minimiert werden und die, v.a. nach der Oberschenkelmarknagelung, erhöhte Rate an Lungenkomplikationen vermieden werden. 
Die Entscheidung zwischen DamageControl-Versorgung oder definitiver Versorgung sollte am Ende der Schockraumversorgung erfolgen. Schaftfrakturen von Ober- und Unterschenkel werden mit Fixateuren versorgt, kniegelenksnahe und sprunggelenksnahe Frakturen mit einem gelenkübergreifenden Fixateur stabilisiert. Problematischer ist die Versorgung von proximalen Femurfrakturen, da gelenkübergreifende Fixateure im Hüftbereich die Lagerungsmöglichkeiten und Pflege deutlich einschränken. Ist eine rasche Stabilisierung des Patienten innerhalb des 1 . Tages absehbar, kann daher eine Lagerung auf einer Schiene oder in Ausnahmefällen die Anlage einer Extension bis zur endgültigen Versorgung ausreichend sein. Bei absehbar längerem Verlauf empfiehlt sich trotz der Nachteile die Anlage eines Fixateur externe.

Wird planmäßig mit einer definitiven Versorgung begonnen, sollte die Reihenfolge jedoch vorher so festgelegt werden, dass bei intraoperativer Verschlechterung des Patientenzustands jederzeit ein sinnvoller Rückzug zur Fixateurversorgung möglich ist.

Der Zustand sollte daher in regelmäßigen Abständen mit dem Anästhesisten kommuniziert werden, um möglichst zeitnah einen Wechsel des Therapieregimes durchführen $\mathrm{zu}$ können. In Abb. 5 ist ein solches mehrzeitiges Vorgehen dargestellt.

Im Falle von kritischen Verletzungen der unteren Extremität mit subtotaler Amputation oder ausgeprägten Gefäß-/Nervenschäden sind bei der Planung der Versorgung die Gesamtverletzungsschwere und der Lokalbefund zu beachten.

Es gilt der Leitspruch „Life before limb“ und die Rekonstruktion der Extremität sollte den Patienten in keinem Fall vital gefährden.

Andernfalls ist eine frühzeitige Amputation vorzuziehen.

\section{Aseptischer Verfahrenswechsel von Fixateur externe auf Marknagelosteo- synthese}

Wie bereits in den topografisch geordneten Abschnitten erwähnt, stellt der Fixateur externe meist keine gute Option zur endgültigen Frakturbehandlung an Ober- und Unterschenkel dar. Insbesondere am Oberschenkel sind erhebliche
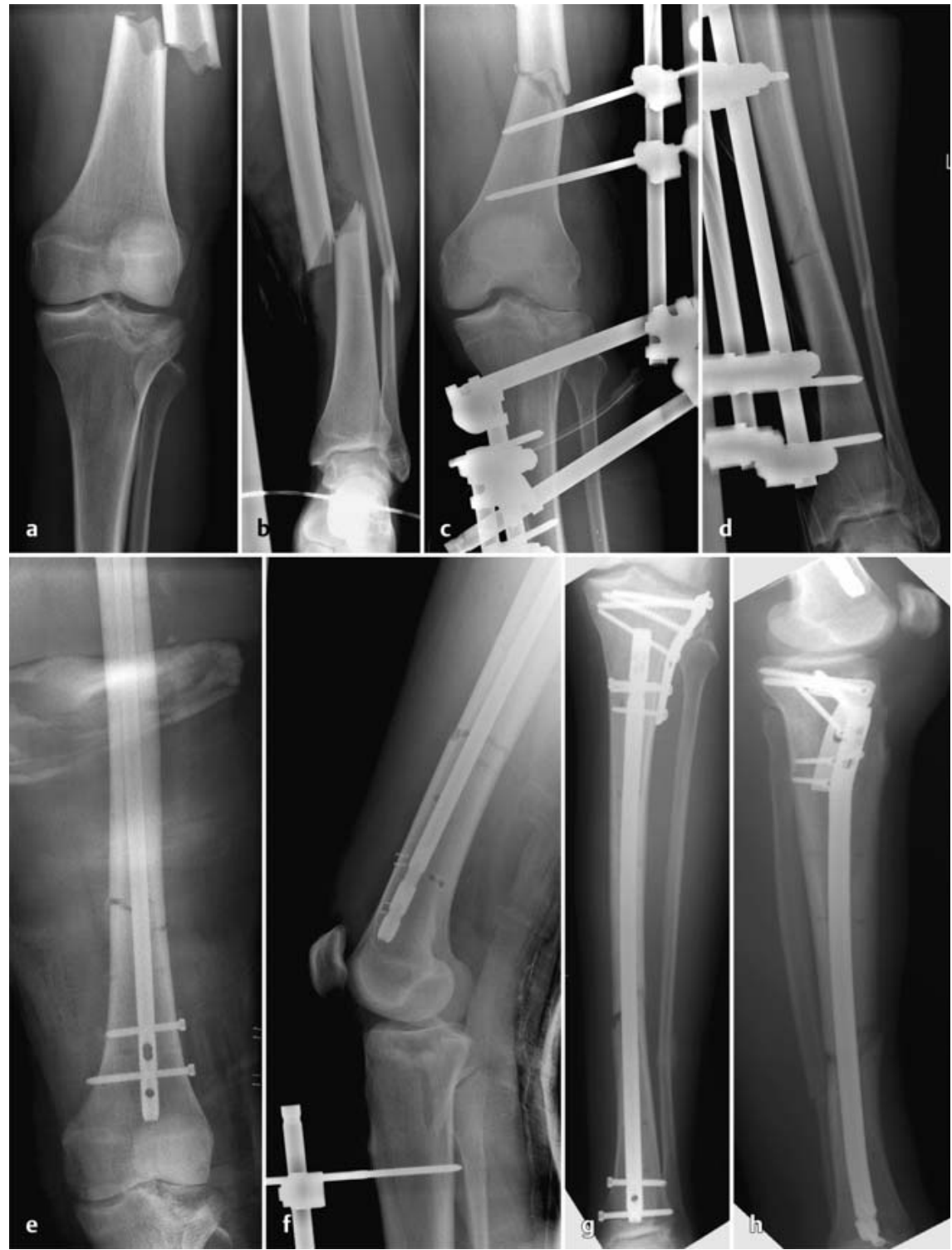

Abb. $\mathbf{5}$ a bis $\mathbf{h}$ Die Röntgenbilder zeigen die Versorgung einer Kettenverletzung (Oberschenkelschaft-, Tibiakopf- und Unterschenkelschaftfraktur) bei einem schwer verletzten Patienten nach Motorradunfall. Zuerst erfolgten eine geschlossene Reposition und Anlage eines gelenkübergreifenden Fixateur externe (c, d). 6 Tage später erfolgte eine Marknagelung des Femurs und Anlage eines Oberschenkelliegegipses zur Protektion des Tibiakopfs. Der Unterschenkelfixateur wurde belassen $(\mathbf{e}, \mathbf{f})$. Weitere 3 Tage später erfolgten eine Plattenosteosynthese mit Spongiosaunterfütterung des Tibiakopfs und in gleicher Sitzung die Marknagelung der Tibia (g, h).

Weichteilirritationen und eine verzögerte Knochenheilung zu erwarten.

Ein geplanter Verfahrenswechsel sollte immer so früh wie möglich durchgeführt werden, da mit zunehmender Liegedauer der Schanz-Schrauben die Kontamination von Eintrittsstelle und Knochen und damit auch das Infektrisiko bei einem Verfahrenswechsel steigt.

Indikationen zum Verfahrenwechsel sind insbesondere:
- Frakturen des Oberschenkels

- deutlich verzögerte oder nicht absehbare Frakturheilung bei anliegendem Fixateur externe

- Immobilisation von Gelenken durch den Fixateur

- persistierende Fehlstellung

Unter Antibiotikaprophylaxe ist bei reizlosen Pinstellen innerhalb der ersten 3 Wochen ein einzeitiger Verfahrenswechsel im Normalfall möglich, am Oberschenkel auch danach. 
Ein manifester Pininfekt stellt eine Kontraindikation zum einzeitigen Wechsel dar. Intraoperativ sollte jedoch auch bei reizlosen Verhältnissen immer ein Abstrich entnommen werden.

Die Pinstellen sollten offen gelassen werden. Bei unsicherer Weichteilsituation kann zumindest im Unterschenkelbereich der Fixateur unter Anlage eines Liegegipses und lokaler Weichteilpflege abgenommen und die Abheilung der Pinstellen vor der definitiven Osteosynthese abgewartet werden. Jedoch besteht hierbei ein erhöhtes Risiko für eine Dislokation und sekundäre Weichteilschädigung bei instabilen Frakturen.

Wenn möglich, sollte vor Beginn der operativen Maßnahmen bei Unterschenkelmarknagelung geprüft werden, ob das Kniegelenk in die benötigte $90^{\circ}$-Beugestellung mobilisiert werden kann.

Ansonsten sind alternative Verfahren in Betracht zu ziehen.

\section{Spezielle Aufklärung}

Der Patient muss auf das erhöhte Infektrisiko mit Möglichkeit der Ausbildung einer akuten bzw. chronischen Osteomyelitis hingewiesen werden.

\section{Lagerung}

Die Lagerung erfolgt entsprechend dem geplanten definitiven Verfahren. Wenn möglich, sollte der Fixateur vollständig entfernt sowie die Pinstellen gründlich gereinigt werden. Anschließend erfolgen die chirurgische Hautdesinfektion und sterile Abdeckung. Hierbei sollten die ehemaligen Pinstellen gezielt steril abgeklebt werden.

\section{OP-Vorbereitung offene Frakturen}

Die Versorgung offener Frakturen stellt einen Notfall dar und sollte daher schnellstmöglich erfolgen, um das Risiko einer Infektion möglichst gering zu halten.
Höhergradig offene Frakturen, die vom Rettungsdienst bereits verbunden wurden, sollten nicht vor Erreichen des OPSaales erneut inspiziert werden. Nach Eröffnung des Verbands im OP sollte zuerst ein Abstrich für die mikrobiologische Testung genommen werden. Anschließend werden die Haare mit dem Haarschneider entfernt und die Haut gründlich gereinigt. Dies sollte unter möglichst aseptischen Bedingungen geschehen. Wir empfehlen daher den Einsatz steriler Handschuhe und Bürsten sowie steriler Spüllösung und Seife. Nach Reinigung der Haut sollten die Handschuhe gewechselt und anschließend die Wunde und ggf. freiliegende Knochen gereinigt werden. Danach sollte die Wunde gründlich mit steriler isotoner Lösung gespült und bis zum OP-Beginn mit einem sterilen Wundverband bedeckt werden. Erst intraoperativ erfolgt eine gründliche Abtragung von zerstörtem Gewebe, gefolgt von weiterer Spülung mit isotoner Flüssigkeit.

Eine Antibiotikaprophylaxe ist aufgrund der zu erwartenden Kontamination bei offenen Frakturen immer zu empfehlen.

\section{Literatur}

${ }^{1}$ Augat P, Buehren V. Modernes Implantatdesign für Osteosynthesen bei vorbestehender Osteoporose. Orthopade 2010; 39: 397-406

2 Bonnaire F, Mende F, Bula P. Sprunggelenkfrakturen. Trauma und Berufskrankheit 2010; 12: 349-359

3 Caspari RB, Hutton PM, Whipple TL et al. The role of arthroscopy in the management of tibial plateau fractures. Arthroscopy 1985; 1: 76-82

${ }^{4}$ Eidelman M, Katzman A. Treatment of complex tibial fractures in children with the taylor spatial frame. Orthopedics 2008; 31: 992

${ }^{5}$ El Zayat BF, Zettl R, Efe T et al. Minimalinvasive Versorgung geriatrischer und osteoporotischer Femurfrakturen mit polyaxial-winkelstabilem Implantat (NCB-DF ${ }^{\circledR}$ ). Unfallchirurg 2010; Epub ahead of print

${ }^{6}$ Endres T, Grass R, Biewener A et al. Vorteile der minimal-invasiven Reposition, Retention und Ilizarov-(Hybrid)Fixation bei Pilon-tibiale-Frakturen unter besonderer Berücksichtigung von C2-/C3-Frakturen. Unfallchirurg 2004; 107: 273-284

7 Gordon JE, Schoenecker PL, Oda JE et al. A comparison of monolateral and circular external fixation of unstable diaphyseal tibial frac- tures in children. J Pediatr Orthop B 2003; 12: 338-345

8 Grass $R$, Biewener A, Rammelt $S$ et al. Die retrograde Verriegelungsnagelosteosynthese distaler Femurfrakturen mit dem distalen Femurnagel (DFN). Unfallchirurg 2002; 105: 298-314

${ }^{9}$ Hansen M, El Attal R, Blum J et al. Tibiamarknagelung mit dem Expert ${ }^{\circledR}$ Tibia Nagel. Oper Orthop Traumatol 2009; 21: 620-635

10 Holmenschlager F, Piatek S, Halm JP et al. Retrograde Marknagelung von Femurfrakturen mit dem langen Nagel. Eine prospektive Studie. Unfallchirurg 2002; 105: 1100-1108

${ }^{11}$ Kobbe P, Tarkin IS, Oberbeck $R$ et al. Verletzungsadaptiertes Vorgehen bei Unterschenkelverletzungen des Schwerverletzten. Z Orthop Unfall 2008; 146: 580-585

12 Krupp RJ, Malkani AL, Roberts CS et al. Treatment of bicondylar tibia plateau fractures using locked plating versus external fixation. Orthopedics 2009; 32: 559

${ }^{13}$ Logters T, Windolf J, Flohe S. Femurschaftfrakturen. Unfallchirurg 2009; 112: 635-650

14 Maier M, Maier-Heidkamp P, Lehnert $M$ et al. Ausheilungsergebnisse konservativ und operativ versorger kindlicher Femurfrakturen. Unfallchirurg 2003; 106: 48-54

15 Maier M, Marzi I. Die elastisch-stabile Marknagelung der Femurfraktur beim Kind. Oper Orthop Traumatol 2008; 20: 364-372

16 Manca M, Marchetti S, Restuccia G et al. Combined percutaneous internal and external fixation of type- $C$ tibial plafond fractures. A review of twenty-two cases. J Bone Joint Surg [Am] 2002; 84 (Suppl. 2): 109-115

17 Pape HC, Tarkin IS. Intraoperative reduction techniques for difficult femoral fractures. J Orthop Trauma 2009; 23: S6-S11

18 Pape HC, Tornetta III P, Tarkin I et al. Timing of fracture fixation in multitrauma patients: the role of early total care and damage control surgery. J Am Acad Orthop Surg 2009; 17: 541-549

${ }^{19}$ Raschke M, Zantop T, Petersen W. Tibiakopffrakturen. Chirurg 2007; 78: 1157-1169

20 Schlickewei W, Boeker T. Kindorientierte Frakturversorgung. Trauma und Berufskrankheit 2010; 12: 335-340

\section{Dr. med. Philipp Lichte}

Assistenzarzt

Prof. Dr. med. H. C. Pape, FACS

Klinikdirektor

Klinik für Orthopädie und

Unfallchirurgie

Schwerpunkt Unfallchirurgie

Universitätsklinikum Aachen

Pauwelsstraße 30

52074 Aachen

plichte@ukaachen.de 